\title{
Análisis vial de la infraestructura logística en la Ciudad de Riobamba
}

\section{Road analysis of the Logistic Infrastructure in the City of Riobamba}

\author{
Miriam del Roció Salas Salazar. ${ }^{1}$, Hernán Vladimir Pazmiño Chiluiza. ${ }^{2}$ \& Juan Enrique \\ Ureña Moreno. ${ }^{3}$
}

\begin{abstract}
.
DOI: https://doi.org/10.33262/concienciadigital.v3i3.1317

Mobility in one of the most important aspects in the economy of a country, accompanied by a logistic infrastructure as in the case of this study, the roads or highways of our provinces that allow the access of vehicles from different provinces, a large amount is evident of cooperatives for internal mobility that is increasingly increasing, this is how the roads in the city of Riobamba are classified in main and secondary streets; The canton must have a mobility plan and at the same time it is essential to have a road ranking plan, established both in the Ecuadorian roads law and in the ordinances of the Riobamba canton, criteria that will allow the determination of express streets, main arterial roads, collectors and premises under technical, geometric characteristics and functionality of the secondary arterial pathways, the objective is to determine the level of service provided. On the other hand, the interprovincial movement of our Province, because it is located in the center of the country, has a large number of vehicles that enter daily, together with this, the interprovincial cooperatives that have an operating permit, control and planning is an emergency for this city work destined to the bodies that have their competence as the Gad.
\end{abstract}

Keywords: Logistics, tracks, organization, transport, market, competitions.

\footnotetext{
${ }^{1}$ Escuela Superior Politécnica del Chimborazo, Facultad de Administración de Empresas Escuela Gestión de Transporte; Riobamba Ecuador, miriam.salas@espoch.edu.ec

${ }^{2}$ Universidad Nacional de Chimborazo, Facultad de Ingeniería Civil, Riobamba Ecuador, vlapch@ hotmail.com

${ }^{3}$ Escuela Superior Politécnica del Chimborazo, Facultad de Ciencias; Riobamba Ecuador, juan.urena@espoch.edu.ec
} 


\section{Resumen.}

La Movilidad en uno de los factores más importantes en la riqueza de un país, acompañado de una infraestructura logística como en el caso de este estudio las vías o vías de nuestra provincias que accede el acceso de vehículos procedentes de diferentes provincias, se evidencia gran cantidad de cooperativas para la movilidad interna que cada vez va en incremento es así como las vías en la ciudad de Riobamba están clasificadas en calles principales y secundarias; cantón debe contar con un plan de movilidad y a la vez es indispensable poseer un régimen de jerarquización vial, determinados tanto en la ley de vías del Ecuador como en un procedimiento de jerarquización vial, sólidos tanto en la ley de vías y caminos del Ecuador como en estatutos del cantón Riobamba, criterios que permitirán determinar calles expresas, arteriales principales, colectoras y locales bajo características técnicas, geométricas y funcionalidad de las vías leyes del cantón Riobamba, criterios que permitirán determinar calles expresas, arteriales principales, colectoras y locales bajo características técnicas, geométricas y funcionalidad de las vías arteriales secundarias, el objetivo es determinar el nivel de servicio brindado.

En permuta, el movimiento interprovincial nuestra Provincia por encontrarse en el centro del país tiene una gran cantidad de vehículos que ingresan diariamente sumado a esto las cooperativas en interprovinciales que poseen permiso de funcionamiento, el control y la planeación es una urgencia para esta ciudad trabajo destinado al organismo que tienen su competencia como las Gad.

Palabras clave: logística, vías, cooperativas, transporte, mercado, competencias.

\section{Introducción.}

La infraestructura logística constituye uno de los aspectos más importantes en la economía del país de aquí parte importancia de movilidad en nuestra ciudad se evidencia en diferentes trabajos presentados e información obtenida por las diferentes instituciones asignadas al control de la misma, se evidencia gran cantidad de cooperativas para la movilidad interna que cada vez va en incremento es así como las vías en la ciudad de Riobamba están clasificadas en calles principales y secundarias; cantón debe contar con un plan de movilidad y a la vez es indispensable poseer un plan de orden jerárquica vial, señalados tanto en la ley de caminos del Ecuador como en reglamentos del cantón Riobamba, criterios que permitirán determinar calles expresas, arteriales principales, colectoras y locales bajo características técnicas, geométricas y funcionalidad de las vías arteriales secundarias, el objetivo es determinar el nivel de servicio brindado.

En cambio, la movilidad interprovincial nuestra Provincia por encontrarse en el centro del país tiene una gran cantidad de vehículos que ingresan diariamente sumado a esto las cooperativas en interprovinciales que poseen permiso de funcionamiento.

La rehabilitación constituye un punto importante en las vías, accesos en la ciudad forma parte de la reactivación económica garantizando el descongestionamiento sobre todo en las zonas más transitadas con énfasis en el mejorando la seguridad vial, movilidad e imagen y de una u otra manera fuentes de empleo. 
La infraestructura vial de la área urbana y rural del cantón Riobamba es uno de los estudios importantes en el servicio brindado a la ciudad con ciertas especificaciones técnicas de diseño y construcción verificando condiciones cómodas y seguras que permita la circulación

La provincia de Chimborazo está conformado por las siguientes parroquias urbanas Lizarzaburu, Yaruquies Maldonado, Velasco, Veloz y Maldonado que conforman el casco urbano y 11 parroquias rurales, a saber: Cacha, Calpi, Cubijes, Químiag, Flores, Lícto, Licán, Pungalá, Punín, San Juan y San Luis, el Cabildo de la ciudad está presidido por un (GADM), sus competitividades otorgadas , con resolución 006-CNC-2012, en rigor a las necesidades propias del territorio cantonal vinculadas al tránsito, transporte terrestre y la seguridad vial.

Tabla 1. Población del Cantón Riobamba por parroquia de Áreas.

\begin{tabular}{lccccc}
\hline Parroquia & Habitantes & $\%$ & Habitantes & $\%$ & Total \\
\hline Cacha & & $0,00 \%$ & 3160 & $100 \%$ & 3160 \\
Cali & & $0,00 \%$ & 6469 & $100 \%$ & 6469 \\
Cubijies & & $0,00 \%$ & 2519 & $100 \%$ & 2519 \\
Flores & & $0,00 \%$ & 4546 & $100 \%$ & 4546 \\
Lican & & $0,00 \%$ & 7963 & $100 \%$ & 7963 \\
Licto & & $0,00 \%$ & 7807 & $100 \%$ & 7807 \\
Pungala & & $0,00 \%$ & 7807 & $100 \%$ & 7807 \\
Punin & & $0,00 \%$ & 5976 & $100 \%$ & 5976 \\
Quimiag & & $0,00 \%$ & 5257 & $100 \%$ & 5257 \\
Riobamba & 146324 & $93,23 \%$ & 10399 & $6.64 \%$ & $156.123 \%$ \\
San Juan & & $0,00 \%$ & 7370 & $100 \%$ & 7370 \\
San Luis & & $0,00 \%$ & 12602 & $100 \%$ & 12602 \\
Total & 146324 & $64,87 \%$ & 79416 & $35,18 \%$ & 225.741 \\
\hline
\end{tabular}

Fuente: INEN 2010.

Por otra fracción, si consideramos la poblacional por sus particularidades étnicas; en el Cantón Riobamba al igual que la provincia posee características similares; según las estadísticas del INEC el $24.24 \%$ de la población se autodefine étnicamente como indígena y el $70.91 \%$ de raza mestiza; sin embargo, es importante resaltar que en la zona de intervención no se producen distingos de raza. (mtop, s.f.)

Las Competencias de las áreas de Transporte terrestre, tránsito y seguridad las cumple el GAD Municipal del Cantón Riobamba desde el 2015, a través de la Dirección de Gestión de Movilidad, Tránsito y Transporte las responsabilidades del cabildo en una demostración en el que se falta de una adecuado orden jerárquico de vías, que desgarran con la continuación de la circulación, esto se recae por la mala colocación del uso del 
suelo, entre comercial, institucional y residencial, provocando dificultades relacionadas a la arbitrariedad de la apropiación del espacio público.

La competencia de ANT( tránsito terrestre, tránsito y seguridad vial )sobre todo en la información en lo referente al inventario del transporte interprovincial certeza a la gran cuantía de vehículos que diariamente circula en nuestra ciudad y provincia

Figura 1. Vías urbanas en la ciudad de Riobamba

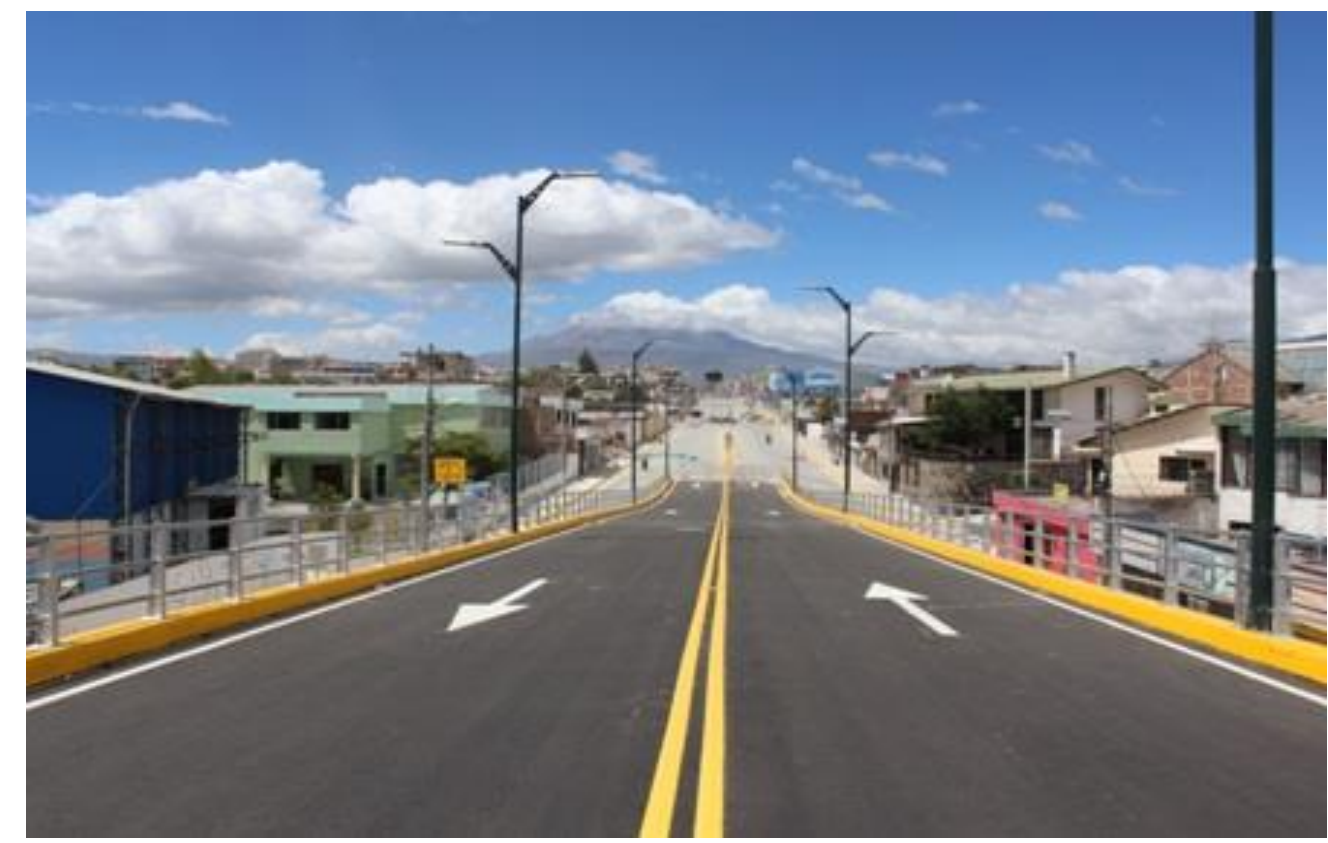

Fuente: Elaboración propia.

\section{Antecedentes Históricos.}

En la cenefa centro del estado se encuentra situada la provincia de Chimborazo, capital a la ciudad de Riobamba, cuyo principio se remonta a la traslación que se realizó desde Cajabamba a la llanura de Tapi después del pasado la sacudida del terremoto de 1797, que destruyo la Ciudad totalmente.

Este nuevo sitio se obligó por una ordenación vanguardista y ordenada en cuadras, amplias calles y avenidas, situación que se le creyera como la ciudad mejor concebida del Ecuador. quedó planificada con reflexiones urbanísticos de observancia, razón que está colocada en un cañón totalmente plano con una progresivo con tendencia de este a oeste, el croquis planificado con vías de norte a sur como calles principales y de vías de este a oeste como calles secundarias se obstaculiza en cada cierto trecho y en lugares poco convenientes, generando aprietos para la partida en el caso de un informe de accidente de tránsito. En la vialidad es el aumento poblacional, con un incremente anual de 1,19\%. El censo población y vivienda reporto para el cantón Riobamba una población de 225.741 habitantes (INEC, 2010), haciendo que el territorio urbano y por ende las vías se difundan rápidamente y sin revisión. 


\section{Marco Teórico.}

En el Ecuador, en la ciudad de Quito, se realizó un "estudio de tráfico en la vía Cumbayá - Pifo (L=15.00km) y propuesta para su mejoramiento" (2012). A lo largo del tramo Cumbayá - Pifo los investigadores establecen o fijan seis puntos de estudio para el análisis de la velocidad de circulación, velocidad de punto y el conteo volumétrico de vehículos., con la finalidad de calcular la capacidad y niveles de servicio actuales, utilizando lo descrito en el Manual de Capacidad de Carreteras (HCM 2000). (2012).

- La Prontitud a la que logran circular los vehículos por las rutas.

- El Turno de recorrido, es decir, la utopía de demoras y esperas.

- Rescate de maniobra con la que los choferes puedan congregar en el tránsito.

- Bienestar que descubren los beneficiarios viales con relación a la excelente situación de la señalética, abandono de ruidos, buen curso de las vías.

- La beneficio o ajuste del flujo a las aspiraciones del usuario.

- Seguridad que mueve y neutra que brinda la vía

Existen los resúmenes tanto internos como externos que afectan el nivel de servicio. Los internos son aquellos que corresponden a diferenciaciones en la velocidad, en el volumen, en la estructura del tránsito, en el porcentaje de movimientos de entrecruzamientos o direccionales, etc. Entre los externos están los tipos físicos, tales como la amplitud de los carriles, el viaje lateral, la anchura de limitaciones, los diferidos, etc.

\section{Costo promedio de muertes por siniestro de tránsito.}

- Costo por fallecido: 200.000 USD

- Pérdida de fuerza Laboral (26 años)

- Indemnización FONSAT

- Gasto Funerario

- Costo Judicial

- Costo Operativos por Emergencia (ANT PLAN NACIONAL DE SEGURIDAD VIAL, 2011)

Pérdida económica por fallecidos desde el Año 2005: 4.000 millones usd

- 20.000 fallecidos por causas asociadas a Siniestros de Tránsito en ese periodo.

Fuente: Dirección de Estudios y Proyectos

Datos: Año 2.011

Fortalecer la Dirección de Estudios y Proyectos, como la dependencia encargada de la Seguridad Vial.

Gestión de seguridad vial constitucional. 


\section{Política 1.1}

Fortalecer la Dirección de Estudios y Proyectos en la gestión de la Seguridad Vial.

\section{Política 1.2}

Suscribir acuerdos y convenios de cooperación con organismos nacionales e internacionales.

\section{Política 1.3}

Regular a través de normas, reglamentos y ordenanzas en coordinación con los diferentes niveles de gobierno. (ANT PLAN NACIONAL DE SEGURIDAD VIAL, 2011)

\section{Respuestas tras accidentes.}

Garantizar una atención integral, oportuna y óptima a las víctimas de los siniestros.

\section{Política 5.1}

Mejorar los sistemas de respuesta inmediata para atención prehospitalaria

\section{Política 5.2}

Incrementar la calidad del servicio en los centros de atención hospitalaria y traumatológica.

\section{Niveles de servicio.}

El Highway Capacity Manual 2000, (Transportation Research Board, 1985) ha establecido seis niveles de servicio denominados A, B, C, D, E y F, que van desde las mejores condiciones de circulación hasta las peores, a continuación, se detallan cada uno de los servicios:

\section{Nivel de servicio A}

Representa una circulación a flujo libre, los usuarios, considerados en forma individual, están virtualmente exentos de los efectos de la presencia de otros en la circulación. Poseen una altísima libertad para seleccionar sus velocidades deseadas y maniobrar dentro del tránsito. El nivel general de comodidad y conveniencia proporcionada por la circulación al conductor, pasajero o peatón, es excelente. (Transportation Research Board, 1985).

\section{Nivel de Servicio B}

Está dentro del clase de flujo estable, aunque se emprenden a observar otros vehículos integrantes del tráfico. La libertad de la selección de las rapideces deseadas sigue comparativamente inafectada, aunque disminuye un poco la libertad de maniobra en relación con la del nivel de servicio A.

El nivel de bienestar y beneficio es algo inferior a los del nivel de servicio A, porque la presencia de otros comienza a influir en el comportamiento individual de cada uno. (Transportation Research Board, 1985) 


\section{Nivel de servicio $\mathbf{C}$}

Pertenece a la clase de flujo estable, pero marca la iniciación del mando en el que la operación de los usuarios particulares se ve afectada de forma explicativa por las interacciones con los otros usuarios. La elección de velocidad se ve afectada por la presencia de otros, y la libertad de maniobra emprende a ser concreta. El nivel de comodidad y conveniencia desciende notablemente. (Transportation Research Board, 1985)

\section{Nivel de servicio $D$}

Simboliza un tráfico de firmeza elevada, aunque estable. La prisa y libertad de artimaña quedan seriamente restringidas, y el conductor o peatón siente un nivel general de comodidad y conveniencia bajo. Los pequeños acrecentamientos de flujo generalmente ocasionan problemas de funcionamiento. (Transportation Research Board, 1985)

\section{Nivel de servicio $\mathbf{E}$}

La maniobra está en él, o cerca del límite de su cabida. La velocidad de todos se ve reducida a un valor bajo, bastante similar. La libertad de artimaña para circular es enormemente difícil, y se consigue forzando a un vehículo o peatón a "ceder el paso". Los niveles de comodidad y beneficio son enormemente bajos, siendo muy elevada la desgracia de los conductores o peatones. EL tráfico es normalmente inestable, debido a que los pequeños aumentos de flujo o ligeras perturbaciones del tránsito producen colapsos. (Transportation Research Board, 1985)

\section{Nivel de servicio $\mathbf{F}$}

Personifica condiciones de flujo obligado. Esta situación se provoca cuando la cantidad de transito que se aproxima a un punto, excede la cantidad que puede desfilar por él. En estas zonas se forman colas, donde la maniobra se caracteriza por la existencia de ondas de parada y arranque, enormemente inestables. (Transportation Research Board, 1985)

Figura 2. 6 niveles de servicio.

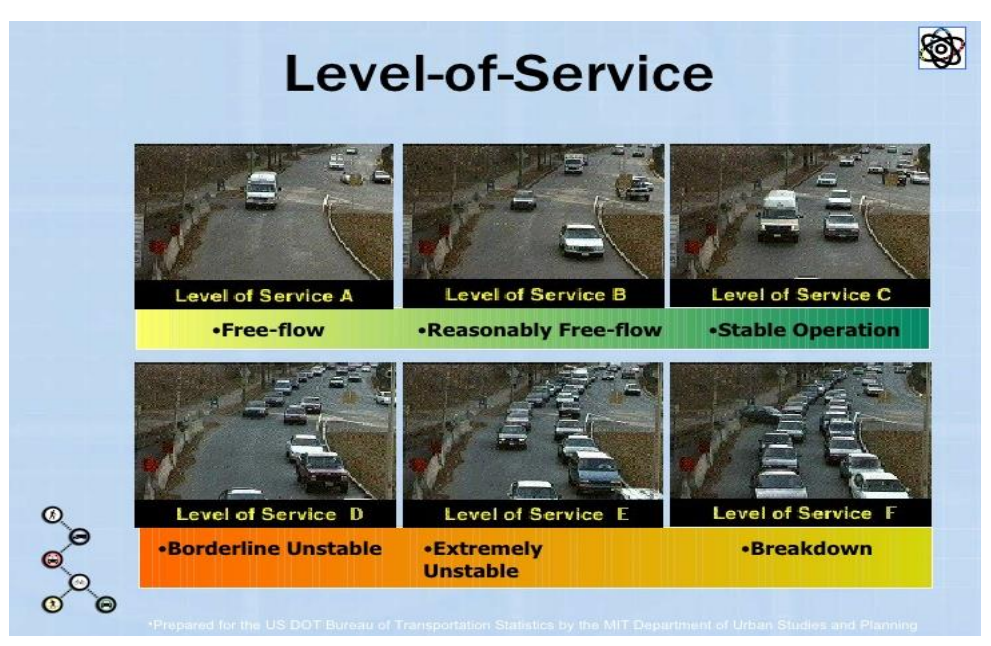

Fuente: (Bond, 2020) 


\section{Software Synchro Traffic.}

La página web Trafficware (2009) rotula las características e importancia de Synchro, es un software de observación y optimización macroscópica. Synchro apoya la 6ta edición, 2010 y 2000 del Highway Capacity Manual (HCM) para enganches no señala, encuentros señalizadas y redondeles. También efectúa la técnica de Uso de Capacidad de Encrucijada para establecer la capacidad de las intersecciones.

\section{Infraestructura vial.}

La Construcción vial es un grupo de compendios que posibilita la circulación de vehículos de forma confortable y segura desde un origen hacia un destino.

La infraestructura vial es el conjunto de componentes físicos que interrelacionados entre sí de manera coherente y bajo el complimiento de viertas especificaciones técnicas de diseño y construcción, ofrecen condiciones cómodas y seguras para circulación de los usuarios que hacen uso de ella. (Montañez, 2016)

La infraestructura vial tiene una importante incidencia en el desarrollo económico de un país y de cada uno de sus cantones dado que contribuye a la mejora de aspectos relevantes como la seguridad y confort de los usuarios viales. Las vías terrestres ayudan a interconectar nodos de consumo y fabricación y el estado de las mismas indica un alto porcentaje del nivel de costos del transporte, los mismos que influyen sobre los flujos de comercialización nacional e universal de un país, por este motivo los proyectos de construcción y mantenimiento de las carreteras necesitan de especial atención de las autoridades pertinentes.

\section{Administración.}

Para (Ortiz, 2009). La administración es "Proceso integral para planear, organizar e integrara una actividad o relación de trabajo, la que se fundamenta en la utilización de recursos para alcanzar un fin determinado".

La administración también puede definirse como la planificación, la dirección, la organización y el control de las actividades dentro de una organización para la cual se requerirá, talento humano, recursos económicos, tecnológicos para alcanzar las metas y poder mantenerse en el mercado competitivo. La administración se aplica para todos los niveles de organización como compañías, cooperativas tales como públicas y privadas con el fin de sacar un mejor rendimiento en términos de eficiencia y eficacia.

\section{Principios generales de la administración.}

Cumplir los principios es una forma más eficiente se necesita que facilite el control, poder realizarlos de una forma inteligente, con experiencia, decisión y mesura. Un principio el cual se requiere es la Coordinación, el cual explica, que se refiere a la forma en que se 
realiza las acciones en la empresa, como pueden ser los problemas en este caso dar las soluciones y no aumentarlos.

\section{De tráfico.}

La ingeniería de transporte deriva de la ingeniería civil y es una profesión interdisciplinaria requerida en cualquier tipo de organización, pública o privada, donde es necesario implementar y administrar sistemas de transporte a nivel nacional e internacional proporcionando soluciones a la problemática que se presenta en los diferentes modos: aéreo, terrestre, ferroviario y marítimo. (Universia, 2013)

Ingeniería de tránsito se define como la rama de la ingeniería que trata del planeamiento de las calles, carreteras, zonas anexas a ellas, del proyecto de sus características geométricas y de la circulación del tránsito en las mismas en vistas a su empleo para transportar personas en forma segura económica y cómoda. (Peña, Herlisseth, 2016)

Seguridades viales el conjunto de mecanismos y acciones que ayuda a garantizar el correcto funcionamiento de la circulación del tránsito, a través de la utilización de conocimientos (reglamento, leyes y disposiciones) y normas de conducta, ya sean estas actuadas como pasajero, peatón o conductor, con el único fin de utilizar de buena forma la vía pública logrando así prevenir siniestros de tránsito. (e-mediadrive, s.f)

\section{Cualitativo.}

Se tomará algunas características del lugar de investigación y los procedimientos que se realizan dentro del lugar de investigación por lo que el investigador realizara la toma la información (Rodriguez \& Valldeoriola, 2009).

\section{De las Garantías para la Habilitación del Suelo}

Artículo. 119. Garantía para la habilitación del suelo. -

Para obtener la correspondiente Licencia Urbanística de habilitación y asegurar que el administrado ejecute el proyecto de habilitación del suelo, la ejecución y entrega de obras a ejecutarse de conformidad con el proyecto técnico aprobado, éste deberá constituir a favor del Gobierno Autónomo Descentralizado del Municipio de Riobamba una garantía de entre las previstas en esta sección, por un monto equivalente al 100\% del valor de las obras a ejecutarse. El administrado asumirá los costos derivados de la obtención de esa garantía.

Se exonera de esta garantía a las subdivisiones y reestructuraciones parcelarias en las que no se requiera la realización de obras.

Artículo. 120. Garantías que puede admitir para la habilitación del suelo. -

El Gobierno Autónomo Descentralizado Municipal de Riobamba aceptará como garantías: hipotecas valoradas por la dependencia encargada de Avalúos y Catastros de lotes desiguales al predio que está en habilitación, prendas, papeles legatarios, garantías bancarias, pólizas de seguros, depósitos en moneda en curso legal, las cuales serán 
entregadas a la Dirección de Gestión Financiera que establecerá las características y los procedimientos específicos para cada caso.

1. Para el caso de hipotecas, se constituirá primera hipoteca sobre inmuebles diferentes al predio a urbanizarse. El administrado asumirá los costos derivados de la obtención de esa garantía.

2. En las urbanizaciones de interés social de desarrollo progresivo efectuadas por organizaciones sociales aprobadas por el Ministerio sectorial competente únicamente servirán como garantía de ejecución de las obras la hipoteca de los lotes.

3. Para el caso del resto de garantías, que igualmente constarán en la Licencia Urbanística, deberán rendirse previa a la obtención de esta, sin que el administrado esté facultado a iniciar las obras de habilitación sin la consignación de aquellas ante la Dirección de Gestión Financiera.

4. Cuando se trate de pólizas de seguros o garantías bancarias, aquellas deberán cumplir como mínimo los siguientes requisitos para que sean consideradas por la Municipalidad:

a. Serán incondicionales, irrevocables y de cobro inmediato con la sola notificación de la Administración Municipal de que el administrado incumplió la obligación principal, en consecuencia, no se admitirán garantías que determinen trámites administrativos previos.

b. Las garantías deberán otorgarse por todo el tiempo que se haya previsto para la ejecución de la obra; o, deberá prever una cláusula de renovación automática hasta que el administrado obtenga el Informe Aprobatorio de finalización de la obra. (FINAL, 2019)

\section{Tipos de Investigación.}

\section{Investigación de campo.}

La Exploración fue notable de campo, ya que la exploración fue obtenida claramente en el entramado vial del cantón Riobamba, en la capital de provincia y en las partidas parroquiales.

\section{Investigación Exploratoria.}

Brinda obtener una aproximación al problema para poder aprender, es decir, acostumbrar con lo que estamos poner en claro, con esto obtenemos averiguación de representación inicial para poder seguir con una indagación más profunda.

\section{Investigación Descriptiva.}

Traeremos de este tipo para poder aclarar, analizar las resultas obtenidos con los instrumentos que manipulemos en la exploración, y plasmarlos para el alcance de todos los aspectos que se trate.

\section{Investigación Explicativa.}


Este tipo de indagación busca donde se causa las dificultades y dar discernimiento de todas las partes que la preparan, dando, terminaciones, definiciones y así acumular de conocimiento del presente trabajo.

\section{Investigación bibliográfica.}

Es irrefutable basada su investigación en documentos, repositorios, páginas Web para poder aumentar las bases de la investigación y lo hará referencia a su respectiva norma (APA, 2016).

\section{Métodos.}

\section{Método Científico.}

Se forma en el arma que permite desplegar nuevos conocimientos que pueden ser justificados científicamente, su cuidado requiere de un forma sistemático y metódico propio del rigor científico, iniciando de la observación, se instituyen los problemas y insuficiencias que se muestran dentro de un fenómeno, para a través de la estudio de otros instrucciones previos presentar posibles procedimientos, para llegar a consecuencias que se trazan a manera de generalidad a través de conclusiones y recomendaciones.

\section{Método Inductivo.}

El plan inductivo inicia en la investigación de escenarios particulares para llegar a la publicación, esto simboliza que el rigor metodológico parte de la cosecha de datos diversos, para llegar a comprobar generalizaciones

En este compromiso de investigación, el método inductivo se utilizó, analizando los varios factores que establecieron el nivel de jerarquización de la red vial del cantón, para establecer el escenario de la red vial urbana del cantón y mostrar los posibles medios para perfeccionar horizonte de servicios de las calles de acuerdo a su aptitud

\section{Método Analítico.}

El estudio tiene como intención descomponer el todo en partes, para prestar atención pormenorizadamente cada una de ellas. En el caso particular de esta investigación se realizó un estudio específico de las vías que componen el andamio vial de la ciudad y de las partidas parroquiales, estableciendo su jerarquización y vocación de servicio y funcionalidad, dando como consecuencia las relaciones efectivas entre ellas para establecer un todo funcional.

\section{Método Sintético.}

En este sentido la indagación se colocó al cálculo de los datos para lograr una estructura funcional del complicado vial urbano del cantón Riobamba y tener los juicios suficientes como para combinar un plan de jerarquización vial, que adecue mejorara la eficacia de servicio de las vías.

Para ejecutar esta automatización las investigadoras se valieron del software Synchro, al cual se nutrió con la base de datos derivada y arrojo los resultados en puesto de los 
lineamientos de la sexta edición del manual de capacidad de carreteras (HCM), logrando establecer de nivel de servicio de la infraestructura vial del cantón Riobamba.

\section{Técnicas.}

\section{Observación directa.}

Esta habilidad admite el acercamiento eficaz con el fenómeno, creando posible la recolección de la averiguación y de las fichas de forma directa.

En caso determinado de esta investigación, se registró la pesquisa en las diferentes calles que forman el esqueleto vial del sector urbano del cantón Riobamba, accediendo, de acuerdo con los datos derivados, tomar fallos para la orden del plan de jerarquización y establecer los juicios más acertados para su aplicabilidad.

Instrumentos.

Los datos registrados en las fichas de observación se obtuvieron con la utilización de los siguientes equipos e instrumental:

GPS Garmim Oregón 750

Flexómetro (50m)

Tacómetro

Computadora

Calculadora

Plano base catastral de la ciudad de Riobamba

Celular

Población y Muestra.

Población.

Tabla 2. Población

\begin{tabular}{cc}
\hline Área Urbana & $\mathbf{9 1 1}$ \\
\hline Área Rural & 208 \\
\hline Total & 1119 \\
\hline
\end{tabular}

Fuente: Plano Base Catastral I. Municipio de Riobamba.

Elaborado por: Grupo de investigación.

\section{Muestra.}

El espacio urbano de las vías norte sur y oeste y relativos el eje vial de las calles Canónigo Av. Daniel León Borja y Av. 10 de agosto que atraviesan la ciudad en sentido.norte - sur y como referente este - oeste la calle Espejo.

Se examinó la mallada calle de los comienzos parroquiales Calpi, Cacha, San Luis, Punín, Lícto, Licán, Flores Químiag, Pungalá, Cubijíes, San Juan, y las entradas principales de la población. 


\section{Resultados.}

La fórmula para el cálculo es la siguiente:

$$
T P D A \text { actual }=\frac{\text { Total tipo vehiculo }}{\text { Volumen de transito para zonas urbanas }(0,10)}
$$

(A) Livianos. - El Instituto Ecuatoriano de Normalización (2002) precisa como livianos a un vehículo de tipo coche u originario de éste, que es trazado para trasladar un máximo de 12 usuarios.

En (Clasificacion vehicular NTE-INEN 2656), se investiga en cuenta las siguientes categorías para este tipo de vehículo.

- Clase L: motocicletas, tricótomos/mototaxi y cuatrimotor.

- Clase M1: sedán, station wagon, hatchback, coupé, convertible, vehículo deportivo utilitario, limusina, minivan.

- $\quad$ Clase M2: van/ furgoneta de pasajeros, microbús

- Clase N1: camioneta, camioneta doble cabina, van de carga/ furgoneta de carga, camión ligero

(B) Pesados. - El Instituto Ecuatoriano de Normalización (2002) precisa como pesados a un vehículo automotor que tiene un peso bruto no mayor a $3860 \mathrm{~kg}$, o que su peso neto sea superior a $2724 \mathrm{~kg}$ y que su área frontal excede de $4,18 \mathrm{~m} 2$.

- Categoría N2: camión pequeño, camión mediano, camión grande, camión pesado y tracto camión

(C) Buses. - Según la Real Academia Española es un vehículo que tiene una gran capacidad de asientos, el mismo que está destinado al transporte de pasajeros por carretera.

En base a la norma (Clasificacion vehicular NTE-INEN 2656), se toman en cuenta las siguientes categorías para este tipo de vehículo.

- Categoría M3: minibús, bus, bus de dos pisos, piso y medio, articulado, biarticulado, trolebús

Procedimiento para establecer las características técnicas de jerarquización vial:

Para el cálculo de la velocidad se lo ejecuta mediante la ayuda de un auto el mismo que accedió determinar la velocidad de operación a la que caminan los vehículos en las vías a ser analizadas. 
De acuerdo con la velocidad de circulación se seleccionó la distancia de visibilidad de paradas tomando en cuenta que ya están estipulados en la Ordenanza 007-2012, GAD Municipal de Riobamba.

El radio mínimo de esquinas se lo obtuvo mediante AutoCad en el que se visualiza el plano catastral del cantón Riobamba que nos facilitó el GADM-R.

El gradiente se lo determina mediante la fórmula de pendiente por lo que se utilizó la siguiente ecuación:

$$
\text { gradiente }=\frac{d v 1-d v 2}{d h} * 100
$$

Donde:

Dv $=$ Distancia Vertical

$\mathrm{Dh}=$ distancia Horizontal

Jerarquización vial y nivel de servicio actual.

Tabla 3. Cuadro resumen de Jerarquización Vial y el nivel de servicio.

\begin{tabular}{|c|c|c|c|c|c|c|}
\hline \multirow{2}{*}{$\begin{array}{c}\text { Jerarquización } \\
\text { vial }\end{array}$} & \multirow{2}{*}{ No. } & Nombre calle & \multicolumn{4}{|c|}{ Nivel de servicio } \\
\hline & & Sentido & $\mathrm{N}-\mathrm{S}$ & $\mathbf{S - N}$ & E-O & O-E \\
\hline \multirow{14}{*}{ Secundarias } & $\mathbf{1}$ & Av. Atahualpa & & & A & A \\
\hline & 2 & $\begin{array}{l}\text { Av. Pedro Vicente } \\
\text { Maldonado }\end{array}$ & B & B & & \\
\hline & 3 & Av. 9 De Octubre & A & A & & \\
\hline & 4 & Av. Edelberto Bonilla & B & A & & \\
\hline & 5 & Av. Héroes de Tapi & $\mathrm{C}$ & $\mathrm{C}$ & & \\
\hline & 6 & Av. Bypass (E35) & & & B & B \\
\hline & 7 & Av. La Prensa & & & $\mathrm{C}$ & B \\
\hline & 8 & Av. Lizarzaburu & $\mathrm{B}$ & $\mathrm{B}$ & & \\
\hline & 9 & $\begin{array}{l}\text { Av. } 11 \text { de Noviembre } \\
\text { (tramo 1) }\end{array}$ & & & B & A \\
\hline & 10 & Av. Canónigo Ramos & $\mathrm{D}$ & B & & \\
\hline & 11 & $\begin{array}{l}\text { Av. Saint Amand Montront } \\
\text { (tramo 1) }\end{array}$ & & & A & A \\
\hline & 12 & Av. Celso Rodríguez & B & B & & \\
\hline & 13 & Madrid & B & A & & \\
\hline & 14 & $\begin{array}{l}\text { Av. Monseñor Leónidas } \\
\text { Proaño (tramo 1) }\end{array}$ & & & B & B \\
\hline \multirow{3}{*}{ Colectoras } & & $\begin{array}{l}\text { Av. } 11 \text { de Noviembre } \\
\text { tramo } 2\end{array}$ & & & B & B \\
\hline & 15 & Ricardo Descalzi & A & & & \\
\hline & 16 & Brasil (tramo 1) & & & B & A \\
\hline
\end{tabular}




\begin{tabular}{|c|c|c|c|c|c|c|}
\hline & 17 & Ayacucho & $\mathrm{C}$ & & & \\
\hline & 18 & Diego de Almagro & & & & $\mathrm{C}$ \\
\hline & 19 & Av. 21 de Abril & & & $\mathrm{B}$ & B \\
\hline & & $\begin{array}{l}\text { Av. Monseñor Leónidas } \\
\text { Proaño (tramo 2) }\end{array}$ & & & B & A \\
\hline \multirow{37}{*}{ Locales } & 20 & Diego de Covio & & & A & $\mathrm{A}$ \\
\hline & 21 & López de Armendariz & & & $\mathrm{A}$ & A \\
\hline & 22 & José de Araujo & & & A & $\mathrm{A}$ \\
\hline & 23 & José de Peralta & A & A & & \\
\hline & 24 & Antonio de Alcedo & $\mathrm{A}$ & A & & \\
\hline & 25 & Diego de Rodríguez & & & $\mathrm{A}$ & $\mathrm{A}$ \\
\hline & 26 & Rio Coca & & & $\mathrm{A}$ & $\mathrm{A}$ \\
\hline & 27 & Rio Bulubulu & $\mathrm{A}$ & A & & \\
\hline & 28 & Manuel Benjamín Carrión & $\mathrm{A}$ & A & & \\
\hline & 29 & Gaspar Zangurima & & & $\mathrm{A}$ & $\mathrm{A}$ \\
\hline & 30 & Padre M. Orozco & & & $\mathrm{A}$ & A \\
\hline & 31 & Joaquín Pinto & & & $\mathrm{A}$ & $\mathrm{A}$ \\
\hline & 32 & Gustavo Vallejo & & & A & \\
\hline & 33 & Manuel Rendón & $\mathrm{A}$ & A & & \\
\hline & 34 & Agustín Cueva & & & $\mathrm{A}$ & \\
\hline & 35 & Agustín Torres Solís & & & $\mathrm{B}$ & $\mathrm{B}$ \\
\hline & 36 & Jacinto González & & & $\mathrm{B}$ & $\mathrm{B}$ \\
\hline & 37 & Duchicela & & & $\mathrm{B}$ & $\mathrm{B}$ \\
\hline & 38 & Manuel Elicio Flor & $\mathrm{A}$ & & & \\
\hline & 39 & Los Cedros & $\mathrm{A}$ & & & \\
\hline & 40 & José de Orozco & & B & & \\
\hline & 41 & Francia & & & & $\mathrm{B}$ \\
\hline & & $\begin{array}{l}\text { Av. Saint Amand Montront } \\
\text { (tramo 2) }\end{array}$ & & & A & B \\
\hline & & Brasil (tramo 2) & & & $\mathrm{A}$ & $\mathrm{A}$ \\
\hline & 42 & Esmeraldas & $\mathrm{B}$ & & & \\
\hline & 43 & Riobamba & & & $\mathrm{A}$ & $\bar{A}$ \\
\hline & 44 & San Andrés & $\mathrm{A}$ & A & & \\
\hline & 45 & Av. Daniel León Borja & $\mathrm{C}$ & $\mathrm{C}$ & & \\
\hline & 46 & 10 de Agosto & $\mathrm{C}$ & & & \\
\hline & 47 & Roma & $\mathrm{A}$ & B & & \\
\hline & 48 & Paris & & & $\mathrm{B}$ & A \\
\hline & 49 & Puruhá & & & $\mathrm{B}$ & $\mathrm{B}$ \\
\hline & 50 & $\mathrm{LaPaz}$ & & & $\mathrm{A}$ & $\mathrm{B}$ \\
\hline & 51 & Bolívar Bonilla & & & $\mathrm{B}$ & $\mathrm{B}$ \\
\hline & 52 & Buenos Aires & & B & & \\
\hline & 53 & Nueva York & & A & & \\
\hline & 54 & Juan Lavalle & & & $\mathrm{B}$ & \\
\hline
\end{tabular}




\begin{tabular}{|c|c|c|c|c|c|c|}
\hline & 55 & Vicente Rocafuerte & & & & $\mathrm{C}$ \\
\hline & 56 & España & & & $\mathrm{C}$ & \\
\hline & 57 & Juan de Dios Martínez & & & & A \\
\hline & 58 & Eugenio Espejo & & & & $\mathrm{B}$ \\
\hline & 59 & Av. Alfonso Chávez & & & $\mathrm{B}$ & A \\
\hline & 60 & Juan de Velasco & & & $\mathrm{C}$ & \\
\hline & 61 & Mariana de Jesús & & & $\mathrm{B}$ & $\mathrm{B}$ \\
\hline & 62 & Joaquín Chiriboga & & & $\mathrm{C}$ & \\
\hline & 63 & Barón de Carondelet & $\mathrm{B}$ & B & & \\
\hline & 64 & Luis Cordovez & $\mathrm{C}$ & $\mathrm{C}$ & & \\
\hline & 65 & Vicente Ramón Roca & $\mathrm{B}$ & $\mathrm{B}$ & & \\
\hline & 66 & Jaime Roldós Aguilera & & & A & A \\
\hline & 67 & Juan Fernández de Recalde & A & A & & \\
\hline & 68 & Av. Félix Proaño & & & $\mathrm{B}$ & $\mathrm{B}$ \\
\hline & 69 & Gaspar de Villarroel & & $\mathrm{C}$ & & \\
\hline & 70 & Miguel Ángel León & & & $\mathrm{B}$ & $\mathrm{B}$ \\
\hline \multirow{11}{*}{ Rurales } & 1 & Licán & $\mathrm{B}$ & $\mathrm{B}$ & & \\
\hline & 2 & Calpi & $\mathrm{C}$ & $\mathrm{C}$ & & \\
\hline & 3 & San Juan & $\mathrm{B}$ & $\mathrm{B}$ & & \\
\hline & 4 & San Luis & & & A & A \\
\hline & 5 & Punín & & & B & B \\
\hline & 6 & Flores & & & A & A \\
\hline & 7 & Lícto & $\mathrm{C}$ & $\mathrm{C}$ & & \\
\hline & 8 & Pungalá & A & A & & \\
\hline & 9 & Cubijíes & B & B & & \\
\hline & 10 & Quimiag & A & A & & \\
\hline & 11 & Cacha & A & A & & \\
\hline
\end{tabular}

Fuente: Jerarquización vial Chesly Guadalupe y Karina Romero

Tabla 4. Inventario de transporte Interprovincial del Canto Riobamba

\begin{tabular}{c|l|c}
\hline & \multicolumn{2}{|c}{ Interprovincial } \\
\hline Cantones & \multicolumn{1}{|c}{ Operadoras } & \multicolumn{1}{|c}{ Cantón } \\
\hline Guamote & Compañía De Transportes Condorazo Cia. Ltda. & Riobamba \\
\hline Guano & $\begin{array}{l}\text { Cooperativa De Transporte De Pasajeros En } \\
\text { Buses Trans Vencedores }\end{array}$ & Riobamba \\
\hline Pallatanga & $\begin{array}{l}\text { Cooperativa De Transporte Interprovincial De } \\
\text { Pasajeros En Buses Unidos }\end{array}$ & Riobamba \\
\hline Penipe & Cooperativa De Transporte Interprovincial Patria & Riobamba \\
\hline Riobamba & $\begin{array}{l}\text { Cooperativa De Transporte Interprovincial San } \\
\text { Lucas De Ilapo }\end{array}$ & Riobamba \\
\hline
\end{tabular}


ISSN: 2600-5859

\begin{tabular}{l|l|c}
\hline & Cooperativa De Transporte Riobamba & Riobamba \\
\hline & $\begin{array}{l}\text { Cooperativa De Transportes De Pasajeros } \\
\text { Ecuador Ejecutivo }\end{array}$ & Riobamba \\
\hline & Cooperativa De Transportes De Pasajeros Sangay & Riobamba \\
\hline & $\begin{array}{l}\text { Cooperativa De Transportes Interprovincial } \\
\text { Chimborazo }\end{array}$ & Riobamba \\
\hline & Cooperativa De Transportes Nuca Llacta & Riobamba \\
\hline & $\begin{array}{l}\text { Cooperativa Interprovincial De Pasajeros En } \\
\text { Buses Colta }\end{array}$ & Riobamba \\
\hline
\end{tabular}

Fuente: Ant Chimborazo

Información peaje San Andrés categoría tráfico promedio anual peaje SAN ANDRES MES TPDA concesión Rumichaca.

- Livianos 2018 enero - diciembre

- Camión 2 ejes 2018 enero - diciembre

- Buses 2 ejes 2018 enero - diciembre 8

- Camión 3 ejes 2018 enero - diciembre

- Buses 3 ejes 2018 enero - diciembre

- Camión 4ejes 2018 enero - diciembre

- Camión 5 ejes 2018 enero - diciembre

68

- Camión 6 ejes 2018 enero - diciembre

- Motos 2018 enero - diciembre

- Exonerados 2018 enero - diciembre

Total

Con los datos mencionados anteriormente podemos visualizar la cantidad de vehículos promedio que ingresan a nuestra provincia por el peaje San Andrés en el periodo Enero diciembre 2018.

Fuente: Elaboración propia.

\section{Conclusiones:}

- El estudio presentado en las que involucraron 70 calles de la ciudad, distribuidas en un $72 \%$ locales, el $21 \%$ vías secundarias y el $7 \%$ calles colectoras, razón a varios factores que se efectúan en cierta mesura para situar en varios porcentajes de clasificación, de acuerdo con los varios lineamientos de la ley de caminos y la ordenanza municipal 007-2012 del CM de 7370 12602. 
- Riobamba, arrojo un resultado del $64,29 \%$ no tienen separación de calzadas (calzada camino empedrado). En nivel de servicio solo se encuentran en las vías Ay B, lo que concluye que la ciudad necesita un plan de jerarquización vial normalizada por las leyes existentes.

- La cantidad de cooperativas de transporte provincial que se movilizan diariamente en la ciudad sumados a estos los vehículos de otras provincias que ingresas requiere un estudio de planificación interna de movilidad

- Si analizamos desde el punto de vista logístico el incremento vehicular que ayuda a la economía de la ciudad va en incremento, pues urge una correcta planificación vehicular.

\section{Recomendaciones.}

- Los Estudios encontrados en el ANT nos demuestran que el parque Automotor de la ciudad va en incremento lo que quiere decir que además de los vehículos pesados encontramos vehículos privados en aumento lo que requiere urgentemente políticas viales las que ayudarían a reducir los tiempos de traslado de un lugar a otro, al analizar este punto podemos calificar un nivel de servicio no satisface al usuario, por lo que urge estudio que tendrá como propósito el mejorar el movimiento en el cantón.

\section{Referencias bibliográficas:}

ANT plan nacional de seguridad vial. (2011).

Armijos , J., Iza, L., \& Quiñonez, C. (2012). Estudio de tráfico en la vía Cumbayá - Pifo $(\mathrm{L}=15.00 \mathrm{~km}$ ) y propuesta para su mejoramiento. (Tesis de pregrado, Universidad Central del Ecuador). Obtenido de http://www.dspace.uce.edu.ec/handle/25000/284

e-mediadrive. (s.f). ¿Qué es seguridad vial? Obtenido de http://culturavial.com/seguridad-vial/que-es-seguridad-vial.html

Bond, A. (15 de 02 de 2020). Slideshare. Obtenido de https://www.slideshare.net/guestd509af/aicp-prep-course-transportationplanning

FINAL, L. I. (2019). www. gab.riobamba. Obtenido de www. gab.riobamba.

Instituto Ecuatoriano de Normalización. (2002). Gestión ambiental, aire, vehículos automotores, límites permitidos de emisiones producidas por fuentes móviles terrestres de gasolina. Obtenido de http://apps.normalizacion.gob.ec/fileserver/2016/nte_inen_2204-2.pdf

Instituto Ecuatoriano de Normalización INEN. (2016). Clasificación vehicular NTEINEN 2656. Obtenido de http://www.normalizacion.gob.ec/wpcontent/uploads/downloads/2016/05/nte_inen_2656.pdf

Montañez, J. (2016). Infraestructura vial. Obtenido de https://es.slideshare.net/JavierMontaez6/infraestructura-vial-62481695

mtop. $\quad$ (s.f.). https://www.obraspublicas.gob.ec/wpcontent/uploads/downloads/2015/08/Literal-k-Proyecto- 
175200000.0000.372337_PROY.pdf.

Obtenido

de

https://www.obraspublicas.gob.ec/wp-

content/uploads/downloads/2015/08/Literal-k-Proyecto-

175200000.0000.372337_PROY.pdf.

Peña, Herlisseth. (2016). Ingeniería de tránsito. Obtenido de https://es.slideshare.net/herlissethsaia/ingenieria-de-transito-59141921

Portugal, C. (s.f.). Tesis de Celia Portugal. Recuperado el 22 de febrero de 2016, de Universidad Técnica Estatal De Quevedo-Tesis De Celia Portugal: https://www.monografias.com/trabajos 75\%20analisis-foda-herramienasplaneacion-estrategica

Rodriguez, D., \& Valldeoriola, J. (2009). Metodología de Investigación. Barcelona: Universitat Oberta de Catalunya.

Trafficware. (2009). Synchro. Obtenido de http://www.trafficware.com/synchro.html

Transportation Research Board. (1985). Highway Capacity Manual. Obtenido de https://es.scribd.com/doc/245680087/Niveles-de-Servicio-Ingenieria-deTransito-y-Desarrollo-Vial

Universia. (2013). ¿Qué hacen los ingenieros de transporte? Obtenido de http://noticias.universia.net.mx/empleo/noticia/2013/05/20/1024544/que-haceningenieros-transporte.html

\section{Ciencia}




\section{PARA CITAR EL ARTÍCULO INDEXADO.}

Salas Salazar, M. del R., Pazmiño Chiluiza, H. V., \& Ureña Moreno, J. E. (2020). Análisis vial de la infraestructura logística en la Ciudad de Riobamba. ConcienciaDigital, 3(3), 293-312. https://doi.org/10.33262/concienciadigital.v3i3.1317

\section{Ciencia \\ LDigital}

El artículo que se publica es de exclusiva responsabilidad de los autores y no necesariamente reflejan el pensamiento de la Revista Conciencia Digital.

El artículo queda en propiedad de la revista y, por tanto, su publicación parcial y/o total en otro medio tiene que ser autorizado por el director de la Revista Conciencia Digital.
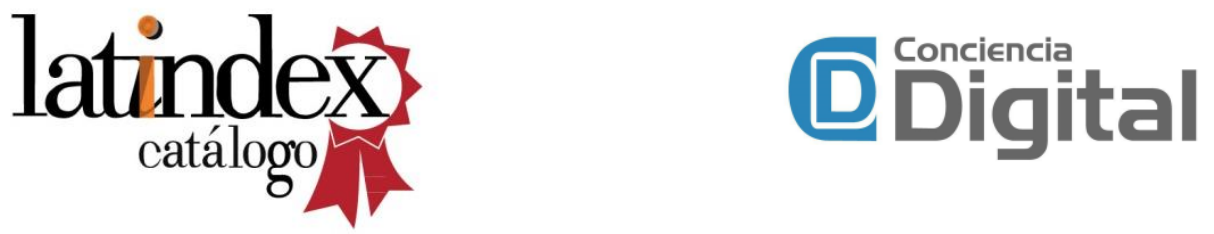